\title{
The optimal duration of anti-tuberculous therapy before pericardiectomy in constrictive tuberculous pericarditis
}

\author{
Likui Fang, Guocan Yu, Bo Ye, Fangming Zhong and Gang Chen ${ }^{*}$
}

\begin{abstract}
Background: It is unclear about the duration of anti-tuberculous therapy before pericardiectomy (DATT) in the patients with constrictive tuberculous pericarditis. This study aims to explore the optimal DATT and its impact on surgical outcomes in these patients.
\end{abstract}

Methods: We retrospectively enrolled 93 patients with constrictive tuberculous pericarditis undergoing pericardiectomy and divided them into two groups according to the optimal cutoff value of DATT which was determined by the receiver operating characteristic (ROC) curve and Youden Index. Postoperative and survival outcomes were compared between the two groups.

Results: The optimal cutoff value of DATT was 1.05 (months). The enrolled patients were divided into the DATT $\leq 1.05$ group and the DATT > 1.05 group, with 24 (25.8\%) and 69 (74.2\%) cases, respectively. Comparing with the DATT $\leq 1.05$ group, the DATT $>1.05$ group had shorter postoperative ICU stay $(P=0.023)$, duration of chest drainage $(P=0.002)$, postoperative hospital stay $(P=0.001)$ and lower incidence of postoperative complications $(P<0.001)$. There were no statistical differences between the two groups in recurrence and survival outcomes.

Conclusions: It would be of potential benefit to enhance recovery after pericardiectomy if DATT lasted for at least 1 month in the patients with constrictive tuberculous pericarditis.

Keywords: Anti-tuberculous therapy, Pericardiectomy, Constrictive tuberculous pericarditis

\section{Introduction}

Although positive public health approaches are implemented in most countries, tuberculosis remains one of the most common infectious diseases and a major cause of death in the world [1, 2]. In developing countries, tuberculosis is the dominant cause of pericarditis [3-5]. Due to the chronic inflammation of tuberculous pericarditis, pericardium becomes fibrotic, thickened and inelastic which inhibits the ventricular filling and eventually

*Correspondence: cgang06@sina.com

Department of Thoracic Surgery, Hangzhou Red Cross Hospital,

Hangzhou 310003, China leads to constrictive pericarditis even after standard antituberculous therapy (ATT) $[6,7]$.

Constrictive tuberculosis pericarditis is associated with poor treatment outcomes of medication therapy $[8,9]$. Surgical intervention is recommended if there is persistent pericardial constriction and pericardiectomy is the standard surgical method to relieve the constriction [9-11]. Although pericardiectomy is regarded as the most effective treatment of constrictive tuberculous pericarditis, it is accompanied with high risk of postoperative complications and mortality [12]. There have been some studies reporting the possible risk factors of poor outcomes after pericardiectomy, but no one focuses on the duration of anti-tuberculous therapy before pericardiectomy (DATT). This study aims to explore the original author(s) and the source, provide a link to the Creative Commons licence, and indicate if changes were made. The images or other third party material in this article are included in the article's Creative Commons licence, unless indicated otherwise in a credit line to the material. If material is not included in the article's Creative Commons licence and your intended use is not permitted by statutory regulation or exceeds the permitted use, you will need to obtain permission directly from the copyright holder. To view a copy of this licence, visit http://creativecommons.org/licenses/by/4.0/. The Creative Commons Public Domain Dedication waiver (http://creativecommons.org/publicdomain/zero/1.0/) applies to the data made available in this article, unless otherwise stated in a credit line to the data. 
optimal DATT and its impacts on surgical outcomes in the patients with constrictive tuberculous pericarditis.

\section{Materials and methods Patients selection}

The records of all patients with constrictive tuberculous pericarditis in our department were retrospectively reviewed from November 2012 to November 2019. The patients who underwent pericardiectomy were included in this study. Finally, a total of 93 patients were enrolled. Their clinical characteristics including DATT were collected from the hospital electronic medical records system. The study protocol was approved by the Institutional Review Board of Hangzhou Red Cross Hospital.

\section{Treatment}

All patients were recommended to receive the standard first-line anti-tuberculous regimen (isoniazid, rifampin, pyrazinamide, and ethambutol) [13], but if patients could not tolerate these drugs, the regimen was adjusted according to individual conditions. Percutaneous internal jugular vein puncture and catheterization were routinely performed preoperatively to monitoring the central venous pressure (CVP).

Median sternotomy was routinely performed in all cases without the use of cardiopulmonary bypass. The extent of pericardiectomy included at least the anterolateral pericardium between the two phrenic nerves, the basal pericardium over the diaphragmatic surface, the pericardium on the great arteries and the pericardium from superior vena cava-right atrium junction to inferior vena cava-right atrium junction [14]. The preoperative and postoperative CT pictures of a patient in this study were presented in Fig. 1. Postoperative complications were defined as the comorbidities that occurred after surgery but did not exist before.

\section{Follow-up}

The follow-up information was obtained from the hospital outpatient clinic records and the missing records were collected by telephone. Postoperative duration of ATT and the survival condition were mainly recorded during the follow-up period. Overall survival (OS) was defined as the time interval between the date of surgery and the date of death or last follow-up. OS was calculated in months. The last follow-up time was April 2020.

\section{Statistical analysis}

The receiver operating characteristic (ROC) curve of DATT was used to predict postoperative complications and the optimal cutoff value of DATT was determined by calculating the Youden Index. The enrolled patients were divided into two groups according to the cutoff value. The measurement data and numeration data of the two groups were statistically analyzed with $t$ test and $x^{2}$ test respectively. Kaplan-Meier method and the log-rank test were performed to analyze the survival impact of DATT. All the above analysis was conducted by SPSS software (version 24.0, IBM SPSS Inc. United States). Statistical significance was set at $P$ value $<0.05$ (All $P$ values presented were 2-sided).

\section{Results}

\section{Optimal cutoff value of DATT}

The result of ROC curve for predicting postoperative complications showed that the area under curve (AUC) of DATT was 0.800 (95\% CI $=0.706-0.893$, $P<0.001)$ (Fig. 2). The result showed that there was significant correlation between DATT and postoperative
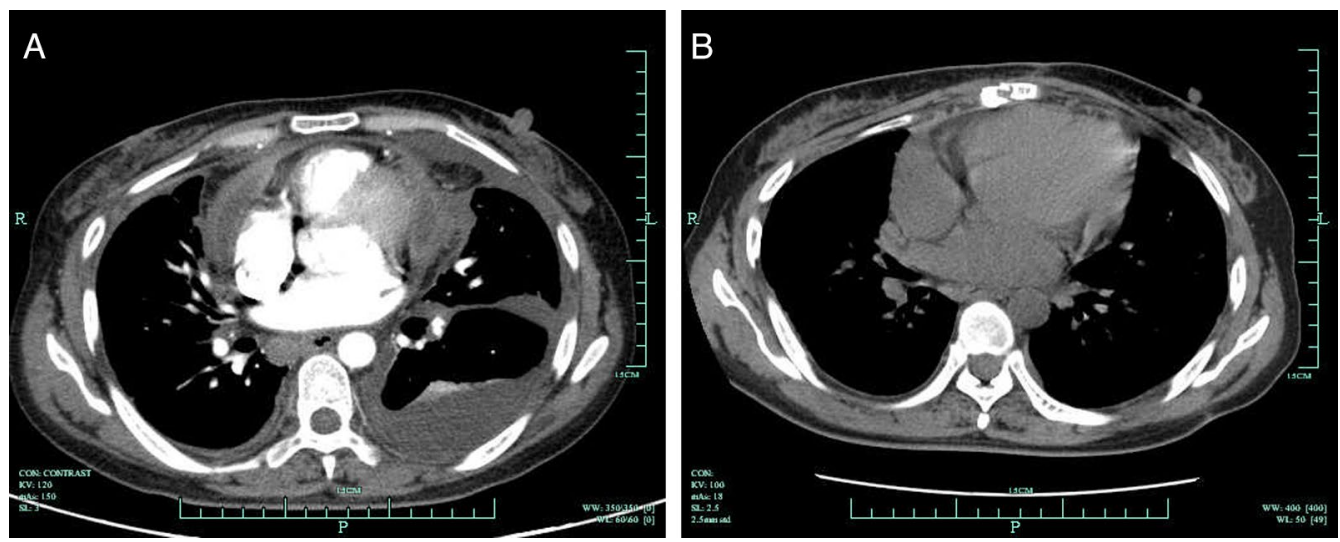

Fig. 1 A The preoperative CT picture of a patient with constrictive tuberculous pericarditis. B The CT picture of the same patient at 2 month postoperatively 


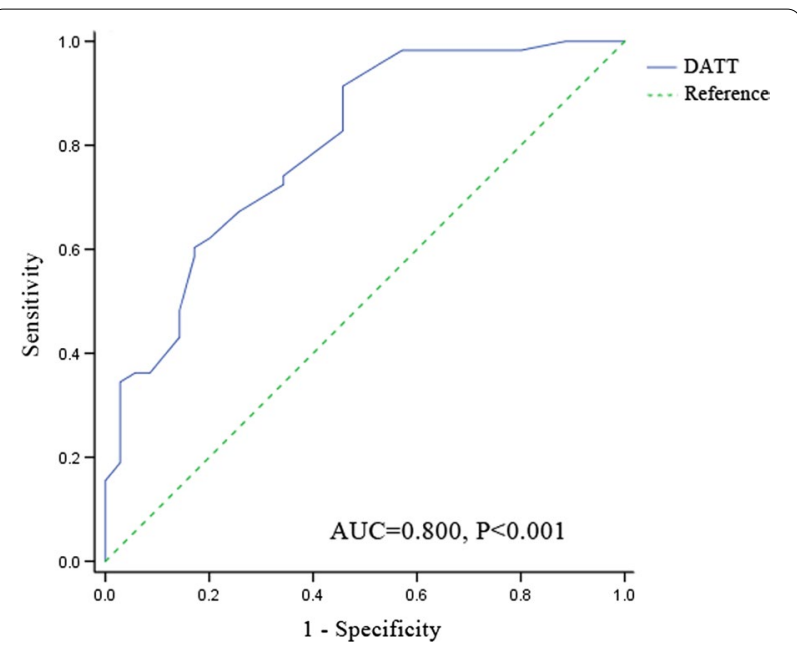

Fig. 2 The area under the $R O C$ curve for postoperative complications determined using DATT. ROC receiver operating characteristic, DATT the duration of anti-tuberculous therapy before pericardiectomy (months), AUC area under the curve

complications. The Youden Index was further calculated and the result showed the optimal cutoff value of DATT was 1.05 months (sensitivity $91.4 \%$, specificity $54.3 \%$, Youden Index 0.457). According to the cutoff value, the patients were divided into the DATT $\leq 1.05$ group and the DATT $>1.05$ group.

\section{Baseline characteristics}

A total of 93 patients were enrolled in the study, with 24 $(25.8 \%)$ cases in the DATT $\leq 1.05$ group and $69(74.2 \%)$ in the DATT $>1.05$ group. The preoperative characteristics of the two groups were presented in the Table 1. There were no statistical differences between the two groups for gender, age, ATT regimens, body mass index (BMI), preoperative central venous pressure (CVP), C-reactive protein (CRP) and other baseline characteristics except erythrocyte sedimentation rate (ESR). The DATT $\leq 1.05$ group had higher ESR than the DATT $>1.05$ group $(P=0.008)$.

\section{Short-term outcomes}

The comparison of outcomes between the DATT $\leq 1.05$ group and the DATT $>1.05$ group was shown in the Table 2. The two groups were comparable in the operative duration $(P=0.865)$, the volume of blood loss $(P=0.120)$ and postoperative duration of ATT $(P=0.292)$. Comparing with the DATT $\leq 1.05$ group, the incidence of postoperative complications was significantly lower in the DATT $>1.05$ group $(P<0.001)$. In addition, the DATT $>1.05$ group had shorter postoperative ICU stay $(P=0.023)$, duration of chest drainage $(P=0.002)$ and postoperative hospital stay $(P=0.001)$ in comparison to the DATT $\leq 1.05$ group. There was no mortality in the two groups within 30 days after pericardiectomy.

\section{Survival impact}

Follow-up information was successfully collected in 88 (94.6\%) patients and 5 (5.4\%) patients were lost to contact after surgery. The median follow-up time was 38 months, ranging from 5 to 89 months. There was no recurrence of constriction from residual pericardium during the follow-up period. The Kaplan-Meier curve and the logrank test showed that there was no difference in the OS between the DATT $\leq 1.05$ group and the DATT $>1.05$ group $(P=0.391)$ (Fig. 3).

\section{Discussion}

Although the overall incidence of constrictive pericarditis has not been investigated, it appears to be relatively rare worldwide [9]. Tuberculosis has remained the dominant cause of constrictive pericarditis in the developing areas with the rate ranging from 23 to $91 \%[15,16]$. Constrictive tuberculous pericarditis should deserve more attention due to its unfavorable outcomes. The current standard anti-tuberculous regimen of first-line drugs (isoniazid, rifampin, pyrazinamide, and ethambutol) achieves satisfactory cure rate in pulmonary tuberculosis [17], but its effect on the constrictive tuberculous pericarditis has been unclear. There have been several studies indicating that the concentration of anti-tuberculous drugs is low in the pericardial space because of poor penetration, especially rifampin and pyrazinamide [18-20]. Due to the inadequate concentration of primary sterilizing effect drugs, the effect of anti-tuberculous treatment on the development of constrictive pericarditis is limited [8], which suggests that constrictive tuberculous pericarditis is chronic and progressive in most cases. As a result, medication therapy is only a palliative and temporary approach while surgical pericardiectomy is the definitive treatment to relive the constriction and improve clinical symptoms [21, 22].

However, pericardiectomy is associated with high morbidity and mortality [12]. In order to reduce the incidence of postoperative complication and death, some studies have attempted to find the potential risk factors to improve the strategies of perioperative management. The extent of pericardial resection was one of the risk factors and complete pericardiectomy was associated with superior surgical outcomes to partial pericardiectomy [16, 23, 24]. Cardiopulmonary bypass was also a predictor of poor prognosis and the 30-day mortality rate might reduce without the use of cardiopulmonary bypass [25]. In our study, all patients were performed complete pericardiectomy without the use of cardiopulmonary bypass and there was no 
Table 1 Characteristics of patients at baseline

\begin{tabular}{|c|c|c|c|}
\hline Variables & DATT $\leq 1.05$ group $(N=24)$ & DATT $>1.05$ group $(N=69)$ & $P$ value \\
\hline Gender & & & 0.742 \\
\hline Male & $18(75.0 \%)$ & $54(78.3 \%)$ & \\
\hline Female & $6(25.0 \%)$ & $15(21.7 \%)$ & \\
\hline Age, years & $64(17-83)$ & $58(16-81)$ & 0.563 \\
\hline ATT regimens & & & 0.198 \\
\hline HRZE & $14(58.3 \%)$ & $50(72.5 \%)$ & \\
\hline Other & $10(41.7 \%)$ & $19(27.5 \%)$ & \\
\hline Preoperative NYHA functional class & & & 0.396 \\
\hline । & $1(4.2 \%)$ & $6(8.7 \%)$ & \\
\hline$\|$ & $5(20.8 \%)$ & $24(34.8 \%)$ & \\
\hline III & $17(70.8 \%)$ & $35(50.7 \%)$ & \\
\hline IV & $1(4.2 \%)$ & $4(5.8 \%)$ & \\
\hline Hypertension & $2(8.3 \%)$ & $12(17.4 \%)$ & 0.461 \\
\hline Diabetes & $3(12.5 \%)$ & $3(4.3 \%)$ & 0.359 \\
\hline Atrial fibrillation & $4(16.7 \%)$ & $11(15.9 \%)$ & 1.000 \\
\hline HIV infection & $0(0 \%)$ & $0(0 \%)$ & / \\
\hline $\mathrm{BMI}, \mathrm{kg} / \mathrm{m}^{2}$ & $19.7(14.9-26.6)$ & $21.1(16.3-33.1)$ & 0.057 \\
\hline Preoperative $\mathrm{CVP}, \mathrm{cmH}_{2} \mathrm{O}$ & $30.0(16.0-42.0)$ & $26.0(15.5-40.0)$ & 0.278 \\
\hline Pleural effusion & $24(100.0 \%)$ & $63(91.3 \%)$ & 0.312 \\
\hline Ascites & $15(62.5 \%)$ & $35(50.7 \%)$ & 0.319 \\
\hline Pericardial effusion & $17(70.8 \%)$ & $55(79.7 \%)$ & 0.370 \\
\hline Pericardial calcification & $5(20.8 \%)$ & $17(24.6 \%)$ & 0.706 \\
\hline Pericardial thickness, $\mathrm{mm}$ & $10.9(5.0-18.9)$ & $10.0(3.4-18.9)$ & 0.148 \\
\hline LVEF, \% & $60.9(51.9-78.0)$ & $57.6(39.9-74.0)$ & 0.147 \\
\hline Hemoglobin, g/dL & $123(94-151)$ & $122(90-167)$ & 0.772 \\
\hline$C R P, m g / L$ & $19.6(1.5-99.1)$ & $16.3(0.8-78.0)$ & 0.206 \\
\hline $\mathrm{ESR}, \mathrm{mm} / \mathrm{h}$ & $36.5(7.0-89.0)$ & $16.5(2.0-105.0)$ & 0.008 \\
\hline Albumin, $\mathrm{g} / \mathrm{L}$ & $31.7(24.8-41.7)$ & $33.0(20.3-48.8)$ & 0.566 \\
\hline Total bilirubin, $\mu \mathrm{mol} / \mathrm{L}$ & $14.6(6.4-66.7)$ & $18.8(3.1-59.2)$ & 0.481 \\
\hline Direct bilirubin, $\mu \mathrm{mol} / \mathrm{L}$ & $8.8(3.2-36.4)$ & $11.3(1.8-50.3)$ & 0.329 \\
\hline Serum creatinine, $\mu \mathrm{mol} / \mathrm{L}$ & $85.1(62.8-116.1)$ & $81.2(44.5-120.6)$ & 0.137 \\
\hline Preoperative BNP, pg/mL & $165(21-961)$ & $169(21-786)$ & 0.575 \\
\hline
\end{tabular}

Values presented as $\mathrm{N}$ (percentage) for categorical variables and median (range) for continuous variables

DATT the duration of anti-tuberculous therapy before pericardiectomy (months), HRZE isoniazid, rifampicin, pyrazinamide and ethambutol, NYHA New York Heart Association, HIV human immunodeficiency virus, $B M I$ body mass index, CVP central venous pressure, $L V E F$ left ventricular ejection fraction (measured on echocardiogram), CRP C-reactive protein, ESR erythrocyte sedimentation rate, $B N P$ brain natriuretic peptide

Table 2 Comparison of outcomes between DATT $\leq 1$ and DATT $>1$ groups

\begin{tabular}{lccc}
\hline Variables & DATT $\leq \mathbf{1 . 0 5}$ group (N=24) & DATT $>\mathbf{1 . 0 5}$ group (N=69) & $\boldsymbol{P}$ value \\
\hline Operative duration, min & $243(157-375)$ & $245(115-400)$ & 0.865 \\
Blood loss, mL & $100(40-300)$ & $200(50-800)$ & 0.120 \\
Postoperative complications & $19(79.2 \%)$ & $16(23.2 \%)$ & $<0.001$ \\
Postoperative ICU stay, days & $3.5(0-8)$ & $2(0-11)$ & 0.023 \\
Duration of chest drainage, days & $16.5(5-32)$ & $10(4-52)$ & 0.002 \\
Postoperative hospital stay, days & $22(7-48)$ & $15(9-60)$ & 0.001 \\
Mortality within 30 days & $0(0.0 \%)$ & $12(0.0 \%)$ & $/$ \\
Postoperative duration of ATT, months & $10(3-18)$ & $12(0)$ & 0.292 \\
\hline
\end{tabular}

Values presented as median (range) for continuous variables and $\mathrm{N}$ (percentage) for categorical variables

DATT the duration of anti-tuberculous therapy before pericardiectomy (months), ICU intensive care unit, ATT anti-tuberculous therapy 


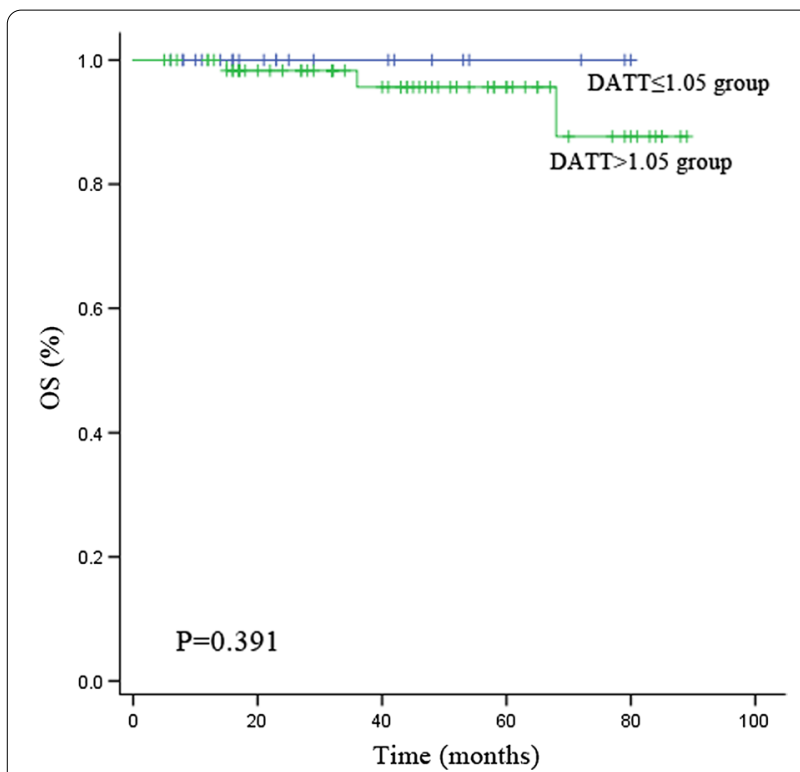

Fig. 3 Kaplan-Meier curves comparing OS between the DATT $\leq 1.05$ group and the DATT $>1.05$ group. DATT the duration of anti-tuberculous therapy before pericardiectomy (months) in-hospital death. In addition to comorbidities, organ functional conditions and etiology of constriction, surgical outcomes were also affected by the timing of pericardiectomy [26, 27]. Early surgical intervention was crucial to reduce mortality and morbidity $[26,28]$. In traditional practice, pericardiectomy was often performed after ATT in constrictive tuberculous pericarditis [8], but the appropriate DATT was unclear.

This study first explored the optimal time interval between the initiation of ATT and pericardiectomy in the patients with constrictive tuberculous pericarditis. We found that 1.05 (months) was the optimal cutoff value of DATT. Shorter postoperative ICU stay and hospital stay were observed in the DATT $>1.05$ group, as well as the duration of chest drainage. In contrast, there was no statistical difference between the two groups in the 30-day mortality after surgery, recurrence and long-term survival. These results indicated that it would be of potential benefit to enhance recovery after surgery if DATT lasted for at least 1 month.

Our study has several limitations. First, this is a single-center retrospective study, so the selection bias is inevitable. Second, due to the relatively small sample size, we only find the lower boundary of DATT but fail to find the upper boundary which requires further studies. Finally, some valuable characteristics such as the extent of pericardial adhesion are failed to be recorded due to the retrospective design.

\section{Conclusion}

It would be of potential benefit to enhance recovery after pericardiectomy in the patients with constrictive tuberculous pericarditis if the duration of anti-tuberculous therapy before surgery lasted for at least 1 month without increasing postoperative recurrence and mortality.

\section{Abbreviations}

DATT: The duration of anti-tuberculous therapy before pericardiectomy; ATT : Anti-tuberculous therapy; HRZE: Isoniazid, rifampicin, pyrazinamide and ethambutol; OS: Overall survival; ROC: Receiver operating characteristic; AUC: Area under curve; NYHA: New York Heart Association; HIV: Human immunodeficiency virus; BMI: Body mass index; CVP: Central venous pressure; LVEF: Left ventricular ejection fraction; CRP: C-reactive protein; ESR: Erythrocyte sedimentation rate; BNP: Brain natriuretic peptide; ICU: Intensive care unit.

\section{Acknowledgements}

Not applicable.

\section{Authors' contributions}

Drs. LF and GC contributed to the conception and design of the work. Drs. LF and GY contributed to data analysis and editing the manuscript. Drs. LF and BY contributed to data acquisition, statistical analysis and interpretation of the data. Drs. GC and FZ contributed to the revision of the manuscript. All authors read and approved the final manuscript.

\section{Funding}

No funding.

\section{Availability of data and materials}

The datasets used and/or analyzed during the current study are available from the corresponding author on reasonable request.

\section{Declarations}

Ethics approval and consent to participate

The study protocol was approved by the Institutional Review Board of Hangzhou Red Cross Hospital.

\section{Consent for publication}

Not applicable.

\section{Competing interests}

The authors declare that they have no competing interests.

Received: 10 December 2020 Accepted: 9 October 2021

Published online: 26 October 2021

\section{References}

1. Furin J, Cox H, Pai M. Tuberculosis. Lancet (London, England). 2019:393(10181):1642-56.

2. Tiberi S, du Plessis N, Walzl G, et al. Tuberculosis: progress and advances in development of new drugs, treatment regimens, and host-directed therapies. Lancet Infect Dis. 2018;18(7):e183-98.

3. Ntsekhe M, Mayosi BM. Tuberculous pericarditis with and without HIV. Heart Fail Rev. 2013;18(3):367-73.

4. Mayosi BM, Ntsekhe M, Bosch J, et al. Prednisolone and Mycobacterium indicus pranii in tuberculous pericarditis. N Engl J Med. 2014;371(12):1121-30.

5. Noubiap JJ, Agbor VN, Ndoadoumgue AL, et al. Epidemiology of pericardial diseases in Africa: a systematic scoping review. Heart (British Cardiac Society). 2019;105(3):180-8. 
6. Dudzinski DM, Mak GS, Hung JW. Pericardial diseases. Curr Probl Cardiol. 2012;37(3):75-118.

7. Jung IY, Song YG, Choi JY, et al. Predictive factors for unfavorable outcomes of tuberculous pericarditis in human immunodeficiency virusuninfected patients in an intermediate tuberculosis burden country. BMC Infect Dis. 2016;16(1):719.

8. Syed FF, Mayosi BM. A modern approach to tuberculous pericarditis. Prog Cardiovasc Dis. 2007;50(3):218-36.

9. Welch TD. Constrictive pericarditis: diagnosis, management and clinical outcomes. Heart (British Cardiac Society). 2018;104(9):725-31.

10. Maisch B, Seferovic PM, Ristic AD, et al. Guidelines on the diagnosis and management of pericardial diseases executive summary; the Task Force on the Diagnosis and Management of Pericardial Diseases of the European Society of Cardiology. Eur Heart J. 2004;25(7):587-610.

11. Adler $Y$, Charron P, Imazio M, et al. 2015 ESC guidelines for the diagnosis and management of pericardial diseases: the Task Force for the Diagnosis and Management of Pericardial Diseases of the European Society of Cardiology (ESC) Endorsed by: The European Association for Cardio-Thoracic Surgery (EACTS). Eur Heart J. 2015;36(42):2921-64.

12. Gopaldas RR, Dao TK, Caron NR, et al. Predictors of in-hospital complications after pericardiectomy: a nationwide outcomes study. J Thorac Cardiovasc Surg. 2013;145(5):1227-33.

13. Zumla A, Raviglione M, Hafner R, et al. Tuberculosis. N Engl J Med. 2013;368(8):745-55.

14. Cho YH, Schaff HV. Extent of pericardial resection for constrictive pericardiectomy. Ann Thorac Surg. 2012;94(6):2180

15. Mutyaba AK, Balkaran S, Cloete $\mathrm{R}$, et al. Constrictive pericarditis requiring pericardiectomy at Groote Schuur Hospital, Cape Town, South Africa: causes and perioperative outcomes in the HIV era (1990-2012). J Thorac Cardiovasc Surg. 2014;148(6):3058-65.

16. Zhu P, Mai M, Wu R, et al. Pericardiectomy for constrictive pericarditis: single-center experience in China. J Cardiothorac Surg. 2015;10:34.

17. Horsburgh CR Jr, Barry CE 3rd, Lange C. Treatment of tuberculosis. N Engl J Med. 2015;373(22):2149-60.

18. Pasipanodya JG, Mubanga M, Ntsekhe M, et al. Tuberculous pericarditis is multibacillary and bacterial burden drives high mortality. EBioMedicine. 2015;2(11):1634-9.
19. Shenje J, Ifeoma Adimora-Nweke F, Ross IL, et al. Poor penetration of antibiotics into pericardium in pericardial tuberculosis. EBioMedicine. 2015:2(11):1640-9.

20. Isiguzo G, Du Bruyn E, Howlett $P$, et al. Diagnosis and management of tuberculous pericarditis: what is new? Curr Cardiol Rep. 2020;22(1):2.

21. Inamdar KY, Aikebaier M, Lijunhong L, et al. Pericardiectomy: prompt surgical management of constrictive pericarditis. Heart Surg Forum. 2014;17(6):E319-22.

22. Syed FF, Schaff HV, Oh JK. Constrictive pericarditis - a curable diastolic heart failure. Nat Rev Cardiol. 2015;12(12):682.

23. Nozohoor S, Johansson M, Koul B, et al. Radical pericardiectomy for chronic constrictive pericarditis. J Card Surg. 2018;33(6):301-7.

24. Choi MS, Jeong DS, Oh JK, et al. Long-term results of radical pericardiectomy for constrictive pericarditis in Korean population. J Cardiothorac Surg. 2019;14(1):32.

25. Rupprecht $L$, Putz C, Florchinger B, et al. Pericardiectomy for constrictive pericarditis: an institution's 21 years experience. Thorac Cardiovasc Surg 2018;66(8):645-50.

26. Lin Y, Zhou M, Xiao J, et al. Treating constrictive pericarditis in a chinese single-center study: a 5-year experience. Ann Thorac Surg. 2012;94(4):1235-40

27. Busch C, Penov K, Amorim PA, et al. Risk factors for mortality after pericardiectomy for chronic constrictive pericarditis in a large single-centre cohort. Eur J Cardio Thorac Surg Off J Eur Assoc Cardio Thorac Surg. 2015:48(6):e110-6.

28. Vistarini N, Chen C, Mazine A, et al. Pericardiectomy for constrictive pericarditis: 20 years of experience at the Montreal Heart Institute. Ann Thorac Surg. 2015;100(1):107-13.

\section{Publisher's Note}

Springer Nature remains neutral with regard to jurisdictional claims in published maps and institutional affiliations.
Ready to submit your research? Choose BMC and benefit from:

- fast, convenient online submission

- thorough peer review by experienced researchers in your field

- rapid publication on acceptance

- support for research data, including large and complex data types

- gold Open Access which fosters wider collaboration and increased citations

- maximum visibility for your research: over $100 \mathrm{M}$ website views per year

At BMC, research is always in progress.

Learn more biomedcentral.com/submissions 